\title{
Atributos da distribuição de carga e indicadores de desempenho logístico: pesquisa com empresas que atuam na região metropolitana de São Paulo
}

\author{
José Geraldo Vidal Vieira, Carla Deguirmendjian Carvalho² e Hugo Tsugunobu Yoshida Yoshizaki ${ }^{3}$
}

\begin{abstract}
Resumo: O objetivo deste artigo é investigar os principais atributos que interferem negativamente no transporte urbano de carga na Região Metropolitana de São Paulo (RMSP) sob o ponto de vista do operador logístico e transportador. Os atributos dizem respeito aos regulamentos para o tráfego e entrega de mercadoria, risco na entrega, colaboração entre as empresas envolvidas no transporte de mercadoria, fatores logísticos e requisitos ambientais. Os indicadores de desempenho logístico adotados por essas empresas também são investigados. Foi realizado um levantamento de dados com 119 representantes de operadores logísticos e transportadoras que atuam na RMSP. Para os respondentes, os atributos que mais dificultam a entrega de mercadoria são congestionamento, roubo de carga e restrição na circulação de veículo. A sazonalidade de entregas durante o mês e as longas filas para carga/descarga foram apontadas pelos operadores logísticos como os maiores problemas; para os transportadores, foram apontados os locais inadequados para carga/descarga e para estacionar veículos de carga. Os indicadores cumprimento do prazo de entrega, atendimento das entregas urgentes, e tempo de entrega na última semana do mês parecem ser os menos afetados pelos atributos de carga.
\end{abstract}

Palavras-chave: logística urbana; transporte urbano de carga; indicadores de desempenho logístico; região metropolitana de São Paulo.

\begin{abstract}
The paper aims to analyze the attributes which negatively impact on urban freight transport, taking into account the viewpoints of logistic service providers (LSPs) and carriers. These attributes are related to regulations for traffic and delivery of goods, risk on delivery, collaboration between such companies involved in the transport of goods, logistics factors and environmental requirements. The Logistics Performance Indicators (LPI) adopted by those companies are also investigated. A survey was carried out with 119 representatives of the LSP and carriers who work in the São Paulo Metropolitan Region (SPMR). According to these representatives, traffic congestion, cargo theft and zone circulation for cargo vehicles are the main issues that hinder the delivery of goods. The seasonality of deliveries during the month and the long queues for loading/unloading were pointed out as the main issues by the LSP; while the lack of adequate places to park and loading/unloading were emphasized by the carriers. Scheduled delivery fulfillment, urgent delivery on time and goods deliveries in the last week of the month seem to be less effected by urban freight transport attributes.
\end{abstract}

Keywords: urban logistics; urban freight transport; logistics performance indicators, São Paulo metropolitan region.

\section{INTRODUÇÃO}

É fato que a distribuição de cargas em grandes centros urbanos é um desafio para as empresas de transporte devido a fatores como falta de local adequado para carga e descarga, restrições de circulação de caminhões, janelas restritas de tempo de entrega, filas, congestionamentos e roubo de carga. Segundo Ogden (1992), a movimentação de carga nas grandes cidades é afetada por problemas estruturais como malha viária deficiente, vias estreitas e congestionamentos causados por carga e descarga nas vias, além de problemas gerados pela falta de estacionamento privado, regulamentação de vagas públicas e áreas específicas para carga e descarga. Esses aspectos negativos podem contribuir para a baixa competitividade das empresas em termos de desempenho logístico. Outros aspectos podem agravar ainda mais a ineficiência operacional na entrega de merca-

\footnotetext{
1 José Geraldo Vidal Vieira, Departamento de Engenharia de Produção, UFSCar. (jose-vidal@ufscar.br)

2 Carla Deguirmendjian Carvalho, Programa de Engenharia de Sistemas Logísticos, USP. (cdcarval@gmail.com)

${ }^{3}$ Hugo Tsugunobu Yoshida Yoshizaki, Programa de Engenharia de

Sistemas Logísticos, USP. (hugo@usp.br)
}

Manuscrito recebido em 22/042015 e aprovado para publicação em 03/06/2015

Este artigo é parte de TRANSPORTES v. 24, n. 4, 2016. ISSN: 2237-1346 (online). DOI:10.14295/transportes.v24i4.912 dorias em grandes centros, como a necessidade de diferentes veículos de carga para atendimento da demanda variada, o aumento da frequência de entregas com um volume menor, a dificuldade de roteirização de entregas, principalmente em locais de difícil acesso, e com restrições de circulação.

Algumas pesquisas sobre distribuição em grandes centros têm sido conduzidas no sentido de investigar normas e atributos que possam melhorar o fluxo de carga urbana, como em Stathopoulos, Valeri e Marcucci (2012); Ballantyne, Lindholm e Whiteing (2013); Lindholm e Behrends (2012). Conforme esses autores, os agentes negligenciam os problemas dos grandes centros e existe pouca colaboração entre eles. Na mesma linha, outras pesquisas foram conduzidas por Antún et al. (2010), Browne et al. (2012), Castro, Kuse e Hyodo (2003), Costabile e Allegrini, (2008), Muñuzuri et al. (2012). Enquanto os governantes focam no transporte público (De Vasconcelos, 2005) e estabelecem normas e restrições de acesso a veículos pesados e em horários de pico (Dablanc, 2007), as empresas focam no desempenho e têm pouca participação nas decisões e planejamentos para os grandes centros (Lindholm, 2013). Esses estudos negligenciaram a investigação de indicadores de desempenho logístico.

No Brasil, os aspectos de distribuição urbana de cargas relacionados ao desempenho logístico das empresas têm sido objeto de estudo por meio de levantamento de dados junto aos principais agentes envolvidos na identificação dos interesses e iniciativas de melhoria de desempenho logístico 
(Dutra, 2004; Sanches Junior, 2008; Moreira, 2012; De Oliveira, 2007; Tacla, Lima Junior, Botter, 2006). No entanto, Sanches Junior (2008) defende que existe uma lacuna de vinte anos com relação aos estudos e pesquisas sobre a distribuição de carga urbana quando comparado com a União Europeia. É fato a não existência de pesquisas sobre logística urbana e desempenho logístico na Região Metropolitana de São Paulo (RMSP) sob a ótica das empresas que atuam na última milha. A abordagem do tema "logística urbana de carga" (Zioni, 2009) e o "desempenho logístico" (Vieira, Yoshizaki, Ho, 2015) das empresas que atuam principalmente na RMSP têm sido conduzida de forma segmentada, com foco em determinados aspectos da distribuição de cargas (Hesse, Rodrigue, 2004).

Portanto, diante da motivação de estudar atributos e indicadores de desempenho logístico numa só pesquisa, o objetivo deste artigo é investigar quais são os principais aspectos de distribuição de cargas que interferem negativamente no desempenho das empresas, sob o ponto de vista das empresas responsáveis pelo transporte de cargas na RMSP (Transportadores e Operadores logísticos). Esses aspectos estão divididos em cinco grupos denominados de atributos: (1) logísticos (uso de sistemas para troca eletrônica de dados, taxa de ocupação de veículo, entrega, fila longa); (2) de colaboração (falta de comprometimento no recebimento de mercadoria pelo cliente, falta de flexibilidade de mudança de horário, falta de confiança na entrega); (3) regulatórios (tráfego intenso, falta de área adequada para estacionamento, carga e descarga); (4) ambientais (incentivo de uso de biocombustível, controle de emissão de $\mathrm{CO}_{2}$ ); e (5) de risco (roubo de carga, enchentes).

Nesta pesquisa, os operadores logísticos são, na sua maioria, empresas de grande porte responsáveis pelo transporte, armazenagem e serviços administrativos relacionados, como gestão dos pedidos, gestão de estoques, planejamento de entrega, monitoramento e rastreamento das entregas, atendimento ao cliente, gestão de reclamações de clientes, gestão de risco e pagamentos (Stefansson, 2006). Os transportadores de porte pequeno a médio são também responsáveis pelo transporte de carga de um ponto ao outro da cadeia de suprimentos, com forte atuação na última milha. Estes transportadores disponibilizam o produto no tempo e local definidos pelo cliente (operador logístico ou embarcador) e podem expandir o escopo de serviços prestados, conforme sua estrutura instalada e capacidade administrativa.

A contribuição acadêmica desta pesquisa se baseia no levantamento de atributos junto à literatura e às empresas que atuam na RMSP. A RMSP é composta por 39 municípios, ocupa uma área de $8 \mathrm{mil} \mathrm{km}^{2}$ (3\% do Estado de São Paulo), com 21 milhões de habitantes e uma frota de 7,5 milhões de veículos (50\% da frota do Estado) (IPEA, 2015; p. 10-15). Estudos em outros centros urbanos também podem se espelhar nesse levantamento à medida que tais atributos podem ser investigados com o objetivo de melhoria do fluxo de mercadorias nesses centros. Adicionalmente, esta pesquisa contribui para as empresas no sentido de mostrar quais indicadores de desempenho logístico podem ser melhorados dada a diversidade de problemas que elas enfrentam para $\mathrm{o}$ atendimento às entregas de mercadorias na RMSP. Para os governantes esta pesquisa também é útil porque dá diretrizes para planejamento e melhoria do acesso às regiões centrais à medida que faz um levantamento das percepções das empresas que distribuem carga na Grande São Paulo. Ressalta-se que esses indicadores se referem às métricas utilizadas pelas empresas para avaliar o desempenho na prestação de serviço ao cliente, referentes ao tempo, espaço, informação, atendimento e confiabilidade. Quanto maior a métrica, melhor o desempenho.

\section{ATRIBUTOS DE DISTRIBUIÇÃO DE CARGA URBANA}

Nesta seção grupos de atributos relacionados à complexidade de carga urbana são descritos. A maioria deles se refere aos regulamentos governamentais e àqueles que envolvem a logística urbana. Allen, Browne e Cherrett (2012) classificam esses atributos em três grupos: (a) Governamentais ou regulatórios - restrição de circulação de veículo de cargas em determinados locais e horários ou por tamanho do veículo, restrição de horário para carga e descarga nas vias, estacionamento de veículo de passeio em vaga para carga e descarga, estacionamento em fila dupla e obstrução do tráfego por veículos de carga e passeio; (b) Gerais - relacionados à operação logística, como definição de janelas de recebimento de mercadoria, frequência de entrega influenciada pela política de estoque pautada em alto giro de estoque e pedido com pequenos volumes, retorno de mercadoria causado por divergência entre pedido e nota fiscal ou falta de pessoal responsável para recebimento, congestionamento, filas e tempo de permanência no cliente durante o processo de carga e descarga, que influenciam o número de viagens diárias e a quantidade de veículos de entrega; (c) Ambientais - como adequação à legislação de emissão de gases poluentes e de efeito estufa, tipo de combustível utilizado.

Ljungberg e Gebresenbet (2005) ao estudarem a cidade de Uppsala, na Suécia, revelaram ineficiências do sistema de entrega devido à alta frequência de entrega de pequenas mercadorias. Essas ineficiências se referem à baixa utilização da capacidade total do veículo identificada no momento da entrega, às elevadas variações no tempo necessário para a entrega das mercadorias e às filas frequentes na área de carregamento.

Cherrett et al. (2012) analisaram 30 pesquisas sobre as atividades do transporte urbano no abastecimento do mercado varejista, considerando taxa média de entrega por semana; número de veículos de entrega por tipo de estabelecimento; taxa de entrega por tipo de cadeia de abastecimento; agendamento de entrega. Os autores também apresentaram alguns estudos relacionados com as características da entrega de mercadoria e o planejamento do transporte, tais como: entrega por dia da semana, tipo de veículo de entrega utilizado, veículos de entrega utilizados na coleta de materiais recicláveis ou devoluções de mercadorias, tempo de permanência durante o carregamento e/ou descarregamento, características da área para carregamento e/ou descarregamento.

Sanches Junior (2008) enfatiza a necessidade de investigar atributos referentes ao uso adequado do solo; característica da carga urbana, como tipo e peso do produto, volume, frequência e horário de carga e/ou descarga; infraestrutura para carga e descarga; número de vagas para carga e/ou descarga; informações sobre o trânsito na cidade; característica do veículo de carga; aspectos ambientais, como nível de ruído emitido nas atividades logísticas, tipo e quantidade de poluentes emitidos; e segurança com base no tipo, quantidade e local de acidentes. 
Anand et al. (2012) classificaram os atributos relacionados à distribuição de carga urbana em cinco categorias:

- Econômica: origem da carga.

- Eficiência: custo do transporte, rotas, fator de carga, emissão de $\mathrm{CO} 2$, fluxo do produto e do tráfego.

- Segurança nas vias: custo do transporte.

- Meio ambiente: nível de poluição, fator de carga, emissão de $\mathrm{CO} 2$, fluxo do tráfego e origem da rota.

- Infraestrutura e gestão: localização, ocupação do solo, fluxo do produto e do tráfego.

O estudo realizado por Browne et al. (2012) aborda os aspectos do transporte de carga que resultam em impactos negativos ao meio ambiente e a economia das cidades (externalidades), tais como congestionamento, poluição sonora e acidentes no trânsito.

Moreira (2012) identificou a incidência de problemas durante as atividades de carga e descarga realizadas na região central da cidade de Belo Horizonte. Essas atividades incluem: (a) características da operação de transporte, como frequência de entrega, número de viagens, baixa eficiência e ocupação do veículo, conferência de carga no recebimento, origem e destino da carga, tipo de produto transportado, adoção de sistemas de monitoramento de carga, quantidade e tipo de veículos utilizados na operação, prazo de entrega, viagem de retorno com caminhão vazio, elevado custo do seguro e frete, demora na carga e descarga, congestionamentos e atrasos na entrega; (b) armazenagem, como tamanho ou instalações inadequadas, falta de segurança contra incêndio, perda e/ou extravio de carga, localização distante dos clientes e/ou fornecedores, falta de equipamento adequado e mão de obra não qualificada; (c) recebimento e despacho de cargas, como a falta de local disponível para carga e descarga, não cumprimento do prazo de entrega, carga danificada, roubo de carga, preço elevado do frete, divergência entre nota fiscal e pedido, veículo inadequado.

Dablanc (1997), em seu estudo realizado em Nova York, Paris e em algumas cidades no norte da Europa, analisou políticas públicas sobre o tema logística urbana e o papel do governo no transporte urbano de carga. A autora destaca alguns aspectos que podem contribuir para um desempenho do transporte urbano de carga eficiente. Os principais aspectos são:

1. Diminuição dos tempos de entrega e possibilidade de entrega noturna.

2. Diminuição de entregas em fila dupla.

3. Diminuição de entregas em vias públicas e, consequentemente, da possibilidade de roubo de carga que esta prática pode ocasionar.

4. Melhor consolidação de carga com maiores taxas de ocupação do veículo.

5. Diminuição das distâncias percorridas entre os pontos de abastecimento, comercial e de consumo.

6. Otimização do roteiro de entregas.

No entanto, essas pesquisas apresentam atributos de forma fragmentada. A proposta deste artigo é investigar esses atributos de distribuição de carga de forma agregada.
Na Tabela 1 é apresentada a síntese dos atributos de distribuição urbana de carga, classificados em: atributos logísticos, atributos regulatórios, atributos ambientais e atributos de risco. Por meio desses grupos distintos, foram investigados os atributos mais citados na literatura ou aqueles que mais contribuem para o contexto brasileiro.

Do total de 49 atributos citados nas pesquisas, temse: 29 atributos logísticos, 10 atributos regulatórios, 5 (cinco) atributos ambientais e 5 (cinco) atributos de risco. Pode-se observar que os atributos logísticos e regulatórios representam aproximadamente $80 \%$ dos atributos citados na revisão de literatura.

Os atributos relacionados à entrega, como a maioria relacionada na Tabela 1, impactam negativamente o trânsito das cidades, por causa de um aumento no número de viagens e de veículos utilizados na movimentação de carga (Taniguchi, Thompson, 2002). O congestionamento das vias compromete o tempo de viagem, o que diminui o número de estabelecimentos atendidos por viagem.

Além do impacto negativo no trânsito da cidade, o aumento de circulação de veículos também é uma das principais fontes de problemas ambientais que os centros urbanos enfrentam atualmente, tais como ruído, consumo de combustível, poluição e acidentes de trânsito (Taniguchi, Thompson, 2002). Por causa disso, as autoridades locais adotam leis ambientais de controle de emissão de gases poluentes e regulamentações sobre o tipo de combustível, o que contribui para a elevação do custo total do transporte de carga para os agentes do setor privado.

Adicionalmente, esta pesquisa englobou os atributos de colaboração, com base em Vieira, Yoshizaki e Ho (2009) e Vivaldini e Pires (2012). Esses atributos se referem à forma como as empresas estabelecem suas relações interpessoais, compartilham informações na gestão da cadeia de suprimentos e definem ações de melhoria em conjunto, buscando o fortalecimento do negócio.

\section{INDICADORES DE DESEMPENHO LOGÍSTICO}

Pires (2011) destaca os indicadores fundamentais que estão relacionados ao resultado global de uma cadeia de suprimentos e são determinantes para a sua competitividade no mercado, como, por exemplo, custos de produção, de estoque e de distribuição, volume de vendas, lucro líquido, prazo de entrega, tempo do ciclo do pedido, além dos indicadores relacionados à qualidade e agilidade no atendimento ao cliente final, tais como entregas no prazo, tempo de resposta, reclamações dos clientes e confiabilidade de entrega.

Allen et al. (2000) sugerem os indicadores de desempenho relevantes na avaliação dos efeitos das políticas de carga urbana adotadas nas cidades de Birgmingham, Basingstoke e Norwick, classificados em operacionais, financeiros e ambientais. Os indicadores operacionais inspiraram a construção dos indicadores de desempenho logístico investigados nesta pesquisa.

Os indicadores operacionais de Allen et al. (2000) são: número de coletas e/ou entregas por viagem, tempo total gasto na viagem por veículo, tempo de deslocamento por coleta e/ou entrega, ocupação do veículo na saída do depósito, relação entre o tempo que o veículo ficou parado para realizar as atividades de coletas e/ou entregas e o tempo 
Tabela 1. Síntese dos atributos relacionados à distribuição de carga urbana

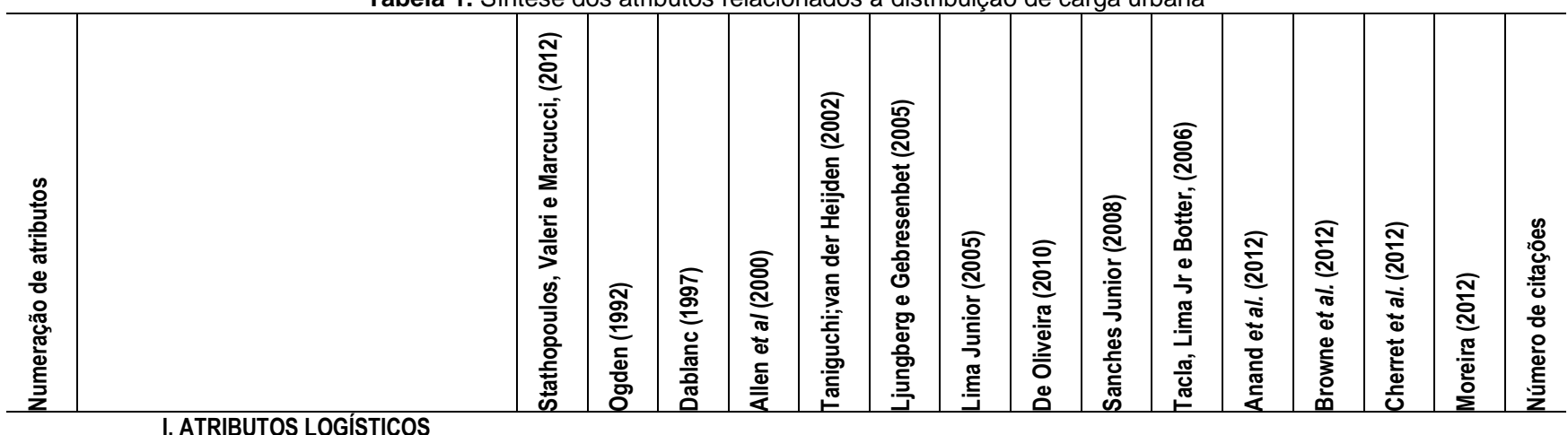

\begin{tabular}{|c|c|c|c|c|c|c|c|c|c|c|c|c|c|c|c|c|}
\hline 1 & Frequência de entrega & & & & $\mathrm{X}$ & & $\mathrm{X}$ & & & $\mathrm{X}$ & & & $\mathrm{X}$ & $\mathrm{X}$ & $\mathrm{X}$ & 6 \\
\hline 2 & Número de viagens & & & & $\mathrm{X}$ & & $\mathrm{X}$ & & $\mathrm{X}$ & $\mathrm{X}$ & & & & & $\mathrm{X}$ & 5 \\
\hline 3 & Número de veículos & & & & $\mathrm{X}$ & & $\mathrm{X}$ & & $\mathrm{X}$ & & & & & $\mathrm{X}$ & $\mathrm{X}$ & 5 \\
\hline 4 & Filas para carga/descarga & $\mathrm{X}$ & & & $\mathrm{X}$ & & $\mathrm{X}$ & $X$ & & & & & & & & 5 \\
\hline 5 & Ocupação do veículo & & & $\mathrm{X}$ & $\mathrm{X}$ & & $\mathrm{X}$ & & & & $\mathrm{X}$ & $\mathrm{X}$ & & & & 5 \\
\hline 6 & Quantidade entregue & & & & $\mathrm{X}$ & & $\mathrm{X}$ & $\mathrm{X}$ & & $\mathrm{X}$ & & & & & & 4 \\
\hline 7 & Entrega noturna & $\mathrm{X}$ & & $\mathrm{X}$ & & & & & $\mathrm{X}$ & & & & & & & 3 \\
\hline 8 & Tipo de veículo & & & & & & & & & $\mathrm{X}$ & & & $\mathrm{X}$ & & $\mathrm{X}$ & 3 \\
\hline 9 & Distância percorrida & $\mathrm{X}$ & & & $\mathrm{X}$ & & & & & $X$ & & & & & & 3 \\
\hline 10 & Retorno de mercadoria & & & & $X$ & & & & $\mathrm{X}$ & & & & & & & 2 \\
\hline 11 & Roteirização & & & & & & & & & & $\mathrm{X}$ & $\mathrm{X}$ & & & & 2 \\
\hline 12 & Tamanho do pedido & & & & $\mathrm{X}$ & & & $\mathrm{X}$ & & & & & & & & 2 \\
\hline 13 & Origem da carga & & & & & & & & & & & $\mathrm{X}$ & & & $\mathrm{X}$ & 2 \\
\hline 14 & Controle de consumo de combustível & & & & & $\mathrm{X}$ & & & & $\mathrm{X}$ & & & & & & 2 \\
\hline 15 & Divergência entre pedido e Nota Fiscal & & & & $X$ & & & & & & & & & & $\mathrm{X}$ & 2 \\
\hline 16 & Capacidade do veículo & & & & & & $\mathrm{X}$ & & & & & & & & & 1 \\
\hline 17 & Falta de equipamento para carga/descarga & & & & & & & & & $\mathrm{X}$ & & & & & & 1 \\
\hline 18 & Falta de pessoal para recebimento & & & & $\mathrm{X}$ & & & & & & & & & & & 1 \\
\hline 19 & Idade do veículo & & & & & & & & & $\mathrm{X}$ & & & & & & 1 \\
\hline 20 & Qualificação da mão de obra & & & & & & & & & & & & & & $\mathrm{X}$ & 1 \\
\hline 21 & Característica da carga & & & & & & & & & $\mathrm{X}$ & & & & & & 1 \\
\hline 22 & Computador de bordo & & & & & & & & & $\mathrm{X}$ & & & & & & 1 \\
\hline 23 & Conferência de carga & & & & & & & & & & & & & & $\mathrm{X}$ & 1 \\
\hline 24 & Conformidade na documentação & & & & & & & & & & & & & & $\mathrm{X}$ & 1 \\
\hline 25 & Destino da carga & & & & & & & & & & & & & & $\mathrm{X}$ & 1 \\
\hline 26 & Frequência de pedido & & & & & & & & & & & & $\mathrm{X}$ & & & 1 \\
\hline 27 & Giro de estoque & & & & & & & $\mathrm{X}$ & & & & & & & & 1 \\
\hline 28 & Monitoramento da carga & & & & & & & & & & & & & & $\mathrm{X}$ & 1 \\
\hline 29 & Número de fornecedores & & & & & & $\mathrm{X}$ & & & & & & & & & 1 \\
\hline \multicolumn{17}{|c|}{ II. ATRIBUTOS REGULATÓRIOS } \\
\hline 1 & Restrição de circulação por tipo de veículo & $\mathrm{X}$ & & & $X$ & & & $\mathrm{X}$ & & & & & $\mathrm{X}$ & & $\mathrm{X}$ & 6 \\
\hline 2 & Vaga para carga/descarga nas vias & $\mathrm{X}$ & $\mathrm{X}$ & & & & & & & & & & $\mathrm{X}$ & & $\mathrm{X}$ & 5 \\
\hline 3 & Restrição de circulação por horário & $\mathrm{X}$ & & & $\mathrm{X}$ & & & $\mathrm{X}$ & & & & & $\mathrm{X}$ & & $\mathrm{X}$ & 5 \\
\hline 4 & Restrição de horário para carga/descarga & $\mathrm{X}$ & & & $\mathrm{X}$ & $\mathrm{X}$ & & & & & & & & & & 3 \\
\hline 5 & Obstrução de vaga de carga/descarga & & $\mathrm{X}$ & & $\mathrm{X}$ & & & & & & & & & & & 2 \\
\hline 6 & Obstrução do tráfego por veículo de carga & & $\mathrm{X}$ & & $X$ & & & & & & & & & & & 2 \\
\hline 9 & Restrição de circulação por final de placa & & & & & & & $\mathrm{X}$ & & & & & & & & 1 \\
\hline 7 & Estacionamento em fila dupla & & & & $\mathrm{X}$ & & & & & & & & & & & 1 \\
\hline 8 & Obstrução do tráfego por veículo de passeio & & & & $X$ & & & & & & & & & & & 1 \\
\hline 10 & Zonas de baixa emissão & & & & & & & & & & & & $x$ & & & 1 \\
\hline \multicolumn{17}{|c|}{ III. ATRIBUTOS AMBIENTAIS } \\
\hline 1 & Tipo de combustível & $\mathrm{X}$ & & & $\mathrm{X}$ & & & & & $\mathrm{X}$ & & & $\mathrm{X}$ & & & 4 \\
\hline 2 & Emissão de poluentes & & & & & $\mathrm{X}$ & & & & $\mathrm{X}$ & & & $\mathrm{X}$ & & & 3 \\
\hline 3 & Emissão de CO2 & & & & & & & & & & & $\mathrm{X}$ & & & & 1 \\
\hline 4 & Ruído & & & & & & & & & & & & $X$ & & & 1 \\
\hline 5 & Coleta de recicláveis ou devolução & & & & & & & & & & & & & $\mathrm{X}$ & & 1 \\
\hline \multicolumn{17}{|c|}{ IV. ATRIBUTOS DE RISCO } \\
\hline 1 & Congestionamento & $\mathrm{X}$ & $\mathrm{X}$ & & $X$ & & & $\mathrm{X}$ & & & $\mathrm{X}$ & & & & & 5 \\
\hline 2 & Roubo ou extravio de carga & & & $\mathrm{X}$ & & & & & & & & & & & $\mathrm{X}$ & 2 \\
\hline 3 & Acidentes de trânsito & & & & & & & & & $\mathrm{X}$ & & & & & & 1 \\
\hline 4 & Número de acidentes com veículo & & & & & & & & & $\mathrm{X}$ & & & & & & 1 \\
\hline 5 & Segurança contra incêndio & & & & & & & & & & & & & & $\mathrm{X}$ & 1 \\
\hline 49 & Total de citações por autor & 9 & 4 & 3 & 21 & 6 & 8 & 8 & 4 & 14 & 3 & 4 & 10 & 3 & 16 & \\
\hline
\end{tabular}


total de viagem, velocidade média por viagem com e sem paradas, distância total percorrida, quantidade de entregas realizadas com veículo estacionado em via pública e em local apropriado para carga e descarga no cliente.

Portanto, os indicadores de desempenho logístico desta pesquisa baseados na literatura são: "entregas no prazo e completas - On Time in Full (OTIF)" (Hesse, Rodrigue, 2004); "cumprimento do prazo de entrega (medido separadamente para aferir se a entrega foi feita no prazo independentemente de sua completude)"; "entregas sem erro", "cargas sem avarias", "entregas urgentes (não planejadas)" (Vieira, Yoshizaki, Ho, 2015); "confirmação de entrega em tempo real" (Munuzuri et al., 2005); "roubo de carga", "devolução de carga", "extravio de carga" (Lu et al., 2003); "trajeto com menor percurso" (Behrends et al., 2008).

\section{METODOLOGIA}

A pesquisa teve caráter descritivo por meio de um levantamento de dados junto ao operador logístico e transportador com o objetivo de analisar os atributos de carga e os indicadores de desempenho logístico. Para tanto, foi feito previamente um estudo de caso em dois operadores logísticos e respectivos parceiros (embarcadores, transportadores e varejistas) com o objetivo de investigar outros indicadores de desempenho logístico que são adotados pelas empresas no dia a dia e também para confirmar aqueles que foram levantados na literatura. Durante o estudo de caso os pesquisadores fizeram entrevistas com representantes das empresas e tiveram acesso aos relatórios e planilhas de acompanhamento dos indicadores de desempenho logísticos e ainda realizaram acompanhamentos de entregas na RMSP junto aos transportadores. Entrevistas por meio de estudos de casos, uso de dados secundários por meio de relatórios e observação direta são procedimentos importantes para a construção de uma pesquisa tipo survey (Forza, 2002). No estudo de caso foram realizadas 38 entrevistas com gerentes/diretores de operações dos dois operadores logísticos e embarcadores, além de acompanhamentos de entrega em 29 pontos de venda com os respectivos parceiros transportadores, com total de $90 \mathrm{~h}$ de trabalho de campo. A escolha dos dois operadores logísticos teve caráter intencional. Um dos operadores logísticos é uma empresa de grande porte e referência internacional na distribuição de carga no varejo. $\mathrm{O}$ operador logístico utiliza, nas operações brasileiras e em outros países, um conjunto de indicadores de desempenho logístico para avaliar suas operações e a de seus transportadores contratados. Este operador logístico tem grande poder na cadeia de suprimento em que atua. Consequentemente, lida com os seus parceiros embarcadores e transportadores diferentemente quando comparado a um pequeno operador logístico, em parte porque adota procedimentos mais padronizados. $\mathrm{O}$ segundo operador logístico é de pequeno porte, de forma a evitar viés na pesquisa. Este operador logístico tinha procedimentos menos formalizados com os seus transportadores e, por outro lado, observou um ambiente mais colaborativo com os seus parceiros.

Após o conjunto de atributos coletados no estudo de caso (operador logístico e respectivos fornecedores de transporte) e na revisão de literatura, foi desenvolvido um questionário (em anexo) o qual foi aplicado aos operadores logísticos e transportadores. Inicialmente, todos os atributos da Tabela 1 foram considerados no pré-teste. Entretanto, somente os mais citados e confirmados pelas empresas foram utilizados na survey. Além disso, outros atributos como "Acúmulo de entregas na última semana do mês", "vias de acessos estreitas", "lei do motorista", "enchentes" e "greves" foram destacados como relevantes pelas empresas do estudo de caso e no pré-teste e, portanto, fizeram parte da pesquisa. Para avaliar os "atributos urbano de carga" os respondentes tiveram que dar uma nota entre 1 (aquele atributo que representasse o menor problema) e 7 (ao atributo que representasse o maior problema para a empresa na distribuição urbana de carga. A mesma escala $(1-7)$ foi usada para avaliar a percepção dos respondentes quanto ao seu desempenho logístico, o valor mais baixo (1) se refere ao indicador com menor desempenho e a nota maior (7) ao de maior desempenho logístico. O "tempo de entrega de mercadorias na última semana do mês" e "disponibilidade de veículos para entrega de mercadorias" foram indicadores que as empresas do estudo de caso declaram como muito importantes para medir o desempenho logístico. Isto ocorre porque há um acúmulo de entregas no final do mês, o que dificulta o atendimento da demanda a um elevado nível de serviço. A disponibilidade de veículo foi declarada importante porque as empresas nem sempre têm veículos disponíveis para atender à demanda, considerando a diversidade de tamanhos, restrições de acesso e alta frequência de entregas diárias.

Para a operacionalização da coleta de dados foi utilizada a plataforma Internet SurveyMonkey e as respostas foram armazenadas em um banco de dados de acesso restrito ao pesquisador. O link da pesquisa foi enviado por e-mail aos representantes de empresas cujos contatos fazem parte de um banco de dados atualizado do Projeto FAPESP 19271-9 e de entidades representativas dos setores envolvidos, a Associação Brasileira dos Operadores Logísticos (ABOL) e o Sindicato dos Transportadores de Carga de São Paulo (SETCESP), no período de setembro a novembro de 2013.

Existem 3.449 transportadoras e 57 operadores logísticos atuando no Estado de São Paulo (Transvias, 2015). No entanto, é difícil determinar o tamanho da população que realiza transporte de carga na RMSP. A unidade amostral é o respondente. $\mathrm{O}$ objetivo é identificar, por meio dos representantes das empresas prestadoras de serviços logísticos, uma visão geral dos problemas de distribuição de carga e os desafios logísticos na RMSP.

Na Tabela 2 é mostrada a taxa de resposta obtida por operador logístico e transportador.

Tabela 2. Taxa de resposta por prestador de serviço logístico

\begin{tabular}{lcccc}
\hline \multirow{2}{*}{ Questionários } & \multicolumn{2}{c}{ Operador logístico } & \multicolumn{2}{c}{ Transportador } \\
\cline { 2 - 5 } & Número & $\mathbf{\%}$ & Número & $\mathbf{\%}$ \\
\hline Total & 43 & 100,00 & 3.449 & 100,00 \\
Respondidos & 30 & 69,76 & 138 & 2,12 \\
Incompletos & 5 & 11,63 & 40 & 0,62 \\
Não considera- & 1 & 0,02 & 3 & 0,05 \\
dos & $\mathbf{2 4}$ & $\mathbf{5 5 , 8 1}$ & $\mathbf{9 5}$ & $\mathbf{1 , 5}$ \\
Válidos & & & &
\end{tabular}


Tabela 3. Medidas descritivas dos atributos de distribuição de carga para o Operador Logístico

\begin{tabular}{|c|c|c|c|c|c|}
\hline ATRIBUTOS & Relevância & Média & Q1 & Md & Q3 \\
\hline \multicolumn{6}{|l|}{ COLABORAÇÃO } \\
\hline Falta de flexibilidade do destinatário para entrega de mercadoria & & 5,54 & 5 & 6 & 7 \\
\hline Falta de comprometimento no recebimento & & 5,21 & 5 & 5 & 6 \\
\hline Falta de confiança no recebimento da mercadoria & & 4,71 & 3 & 5 & 6 \\
\hline Falta de compartilhamento de informação com o cliente & & 4,63 & 3 & 5 & 6 \\
\hline \multicolumn{6}{|l|}{ LOGÍSTICOS } \\
\hline Acúmulo de entregas na última semana do mês & 1 & 6,08 & 6 & 7 & 7 \\
\hline Longas filas para carga e descarga & 1 & 5,83 & 5 & 6 & 7 \\
\hline Vias de acessos estreitas para entrega & & 5,25 & 4,75 & 6 & 7 \\
\hline Necessidade de entrega Noturna & & 5,21 & 4 & 6 & 7 \\
\hline Exigências de equipe para o atendimento (ajudantes) & & 5,21 & 4 & 5 & 7 \\
\hline Exigências para entrega (condições do veículo, equipamento) & & 5,04 & 4 & 5 & 6 \\
\hline Falta tecnologia de informação & & 5,04 & 4 & 5 & 6 \\
\hline Necessidade de diferentes tipos de veículo & & 4,83 & 4 & 5 & 6 \\
\hline Falta treinamento de pessoal de transporte & & 4,67 & 3,75 & 5 & 5,25 \\
\hline Baixa taxa de ocupação & & 4,58 & 3 & 5 & 6 \\
\hline \multicolumn{6}{|l|}{ REGULATÓRIOS } \\
\hline Rodízio de veículos & 1 & 5,83 & 5 & 6,5 & 7 \\
\hline Zona máxima de restrição & 1 & 5,79 & 5,75 & 7 & 7 \\
\hline Local inadequado para carga/descarga & & 5,79 & 6 & 6 & 7 \\
\hline Local inadequado para estacionar o veículo de carga & & 5,63 & 4,75 & 6 & 7 \\
\hline Lei do motorista & & 4,29 & 3 & 5 & 5,25 \\
\hline \multicolumn{6}{|l|}{ AMBIENTAIS } \\
\hline \multicolumn{6}{|l|}{ ATRIBUTOS } \\
\hline Atendimento ao controle de emissão de poluentes & 0 & 4,04 & 2,75 & 4 & 5,25 \\
\hline Atendimento às metas de redução de emissão de $\mathrm{CO} 2$ & 0 & 3,92 & 2,75 & 4 & 5,25 \\
\hline Adequação ao uso de biocombustível & 0 & 3,75 & 2 & 4 & 5 \\
\hline \multicolumn{6}{|l|}{ RISCO } \\
\hline Congestionamento & 1 & 6,42 & 6 & 7 & 7 \\
\hline Roubo de carga & 1 & 5,88 & 5 & 7 & 7 \\
\hline Enchentes & 0 & 4,13 & 2,75 & 4 & 5,25 \\
\hline Greves e manifestações públicas & 0 & 4,08 & 2,75 & 4 & 5 \\
\hline
\end{tabular}

Um total de 24 questionários válidos para operador logístico e 95 questionários válidos para transportador foram obtidos. Aproximadamente um terço dos respondentes dos transportadores não concluíram o preenchimento do questionário. Além disso, quatro questionários foram excluídos da base de dados, pelos seguintes motivos: 1) o respondente não trabalha no setor de logística da empresa, ou 2) trabalha em empresa de comércio eletrônico.

Os resultados do levantamento de dados correspondentes aos 119 questionários válidos coletados neste trabalho foram tabulados e a análise estatística descritiva dos dados da pesquisa está descrita a seguir.

A amostra é composta por executivos que ocupam, na sua maioria, posições de alta gerência, sendo $83,33 \%$ representantes do operador logístico e 78,95\% representantes do transportador, com tempo médio no cargo de 9 anos (Operador logístico) e 14 anos (Transportador).

Os setores de atuação (alimentos e bebidas, automotivo e autopeças, vestuário e acessórios, bazar e material de escritório, telecomunicações e energia, higiene pessoal e limpeza, química, hospitalar e farmacêutico) somam $99 \%$ da amostra dos operadores logístico e $90 \%$ dos transporta- dores. A amostra desses setores revela uma boa variabilidade dos dados, pois estão bem distribuídos em torno de $10 \%$ de representatividade.

Verifica-se que $98 \%$ das transportadoras são empresas de capital nacional e $98 \%$ delas estão localizadas na RMSP, enquanto os operadores logísticos apresentam metade das empresas de capital nacional e 55\% deles estão sediados fora da RMSP.

Quanto ao faturamento, a amostra representa operadores logísticos com faturamento bruto superior a $\mathrm{R} \$ 2,4$ milhões em 2013 e com quadro de lotação superior a 100 funcionários. Cerca da metade dos transportadores faturou menos do que R $\$ 2,4$ milhões no mesmo período; $12,63 \%$ das empresas contrataram menos de 10 funcionários. Isso revela que as transportadoras representam empresas de porte pequeno e médio e os operadores logísticos de porte grande, segundo a classificação do Sebrae (2013).

O percentual de entregas na RMSP é maior do que $50 \%$ para menos da metade dos operadores logísticos, ao passo que $63,16 \%$ dos transportadores realizam mais de $50 \%$ das suas entregas na RMSP. No entanto, $70,83 \%$ dos operadores logísticos atendem mais de 30 pontos de venda 
Tabela 4. Medidas descritivas dos atributos de distribuição de carga para o Transportador

\begin{tabular}{|c|c|c|c|c|c|}
\hline ATRIBUTOS & Relevância & Média & Q1 & Md & Q3 \\
\hline \multicolumn{6}{|l|}{ COLABORAÇÃO } \\
\hline Falta de flexibilidade do destinatário para a entrega & & 5,74 & 5 & 6 & 7 \\
\hline Falta de comprometimento no recebimento & & 5,58 & 5 & 6 & 7 \\
\hline Falta de compartilhamento de informação com o destinatário & & 5,02 & 4 & 5 & 6 \\
\hline Falta de confiança no recebimento da mercadoria & & 4,64 & 3 & 5 & 6 \\
\hline \multicolumn{6}{|l|}{ LOGÍSTICOS } \\
\hline Longas filas para carga e descarga & & 5,94 & 5 & 6 & 7 \\
\hline Acúmulo de entregas na última semana do mês & & 5,44 & 4 & 6 & 7 \\
\hline Vias de acesso estreitas para entrega & & 5,29 & 4 & 6 & 7 \\
\hline Exigências de equipe para atendimento (ajudantes) & & 5,22 & 4 & 6 & 7 \\
\hline Necessidade de diferentes tipos de veículo & & 5,18 & 4 & 6 & 7 \\
\hline Exigências para entrega (condições do veículo, equipamento) & & 4,98 & 4 & 5 & 7 \\
\hline Necessidade de entrega Noturna & & 4,93 & 4 & 5 & 7 \\
\hline Baixa taxa de ocupação & & 4,86 & 4 & 5 & 6,5 \\
\hline Falta tecnologia de informação & & 4,69 & 4 & 5 & 6 \\
\hline Falta treinamento de pessoal de transporte & & 4,44 & 3 & 5 & 6 \\
\hline \multicolumn{6}{|l|}{ REGULATÓRIOS } \\
\hline Local inadequado para carga/descarga & 1 & 6,03 & 6 & 7 & 7 \\
\hline Local inadequado para estacionar o veículo de carga & 1 & 5,99 & 6 & 7 & 7 \\
\hline Zona máxima de restrição & 1 & 5,83 & 5 & 7 & 7 \\
\hline Rodízio de veículos & 1 & 5,53 & 4.5 & 7 & 7 \\
\hline Lei do motorista & 0 & 4,44 & 3,5 & 4 & 6 \\
\hline \multicolumn{6}{|l|}{ AMBIENTAIS } \\
\hline Adequação ao uso de biocombustível & 0 & 4,40 & 3 & 4 & 6 \\
\hline Atendimento ao controle de emissão de poluentes & 0 & 4,39 & 3 & 4 & 6 \\
\hline Atendimento às metas de redução de emissão de $\mathrm{CO}_{2}$ & 0 & 4,31 & 3 & 4 & 6 \\
\hline \multicolumn{6}{|l|}{ RISCO } \\
\hline Congestionamento & 1 & 6,48 & 7 & 7 & 7 \\
\hline Roubo de carga & 1 & 5,96 & 6 & 7 & 7 \\
\hline Enchentes & & 4,80 & 4 & 5 & 7 \\
\hline Greves e manifestações públicas & & 4,73 & 3 & 5 & 7 \\
\hline
\end{tabular}

por dia na RMSP, enquanto esse mesmo número de estabelecimentos é atendido por menos da metade dos transportadores, 48,94\%.

Logo, o número de veículos utilizado diariamente também é maior para o operador logístico, 66,67\% dessas empresas utilizam mais de 10 veículos por dia (na maioria leves e de diversas capacidades) na distribuição de carga na RMSP, enquanto 51,58 \% dos transportadores operam com essas quantidades de veículos. Ainda, $87,50 \%$ dos operadores logísticos contratam mais de $25 \%$ da frota de veículos utilizados na distribuição de carga na RMSP, sendo esse nível de terceirização da frota foi adotado por $57,89 \%$ dos transportadores.

Para a análise dos atributos referentes aos problemas de distribuição de carga na RMSP e indicadores de desempenho logístico, foram utilizadas técnicas estatísticas descritivas univariadas, como medidas de posição e dispersão, apresentadas em forma de tabelas.

\section{APRESENTAÇÃO E ANÁLISE DOS DADOS}

Esta seção está subdividida em duas partes. A primeira parte mostra a avaliação (percepção) das empresas quanto aos problemas referentes à distribuição de carga, e a segunda parte trata dos indicadores de desempenho logístico. Em ambas as partes, medidas descritivas foram utilizadas para mensurar a percepção do Operador logístico e Transportador. Neste caso, medidas de posição, como média, primeiro quartil (Q1), mediana (Md) e terceiro quartil (Q3) foram adotadas. A mediana é uma importante medida central para análise de dados quando se tem uma alta variação do desvio padrão. Neste caso, em todas as tabelas a seguir percebe-se que para a maioria das variáveis a média está muito próxima da mediana, portanto a média foi escolhida para a classificação. 


\subsection{Análise dos atributos de distribuição urbana na RMSP}

As Tabelas 4 e 5 apresentam as medidas descritivas dos atributos que, na visão do Operador logístico e Transportador, respectivamente, representam um menor ou maior problema na distribuição urbana de carga. Os atributos com baixo grau de relevância são indicados por "Zero" e os de maior grau por "Um".

Pela Tabela 4, observa-se que "consumo sazonal (última semana do mês)", "filas para carga e descarga", "rodízio de veículos", "zona máxima de restrição, "congestionamento" e "roubo de carga" (com grau 1) são os que apresentam os maiores problemas para as operações dos operadores logísticos dentro da RMSP. Para esses atributos foram obtidas médias próximas ou maior que 6; mediana 6,5 (ou seja, pelo menos $50 \%$ dos respondentes declararam esse atributo como um grande problema) e pelo menos $25 \%$ dos respondentes $(\mathrm{Q} 3)$ atribuíram nota máxima a eles. Adicionalmente, destacam-se também entre os principais problemas para essas empresas a "falta de local para carga e descarga", "a falta de local para estacionar o veículo", "falta de flexibilidade do destinatário para entrega de mercadoria"; "falta de comprometimento no recebimento" e "vias de acessos estreitas para entrega". Todos esses atributos tiveram Q1 maior ou igual a 4,75; ou seja, $75 \%$ dos respondentes percebem esses atributos como problemas na distribuição de carga. Por outro lado, "enchentes", "greves e "manifestações públicas" e os atributos ambientais não parecem ser problemas para os operadores logísticos, pois, pela mediana $(\mathrm{Md})$, até $50 \%$ dos respondentes deram notas menores ou igual a 4 e pelo menos $25 \%$ atribuíram notas baixíssimas (como revela o Q1).

Analogamente, para os transportadores observa-se, pela Tabela 5, "falta de local para carga e descarga", "falta de local para estacionar o veículo", "zona máxima de restrição" e "rodízio de veículos", "congestionamento" e "roubo de carga (zona de risco)" são os principais problemas na RMSP, como revela as altas notas para média e Md. Os problemas como "filas para carga e descarga", "falta de flexibilidade para entrega em outro horário", "a falta de comprometimento no recebimento de mercadorias" também são percebidos como críticos pelas transportadoras, já que apresentam médias próximas de 6 , medianas com nota 6 e Q1 maior igual a 5. Por outro lado, "lei do motorista" e também os atributos de adequação ao meio ambiente não parecem ser os principais problemas para os transportadores. As análises das medidas de posição desses atributos revelam valores similares aos dos operadores logísticos, com destaque para as menores medianas. Ou seja, até $50 \%$ dos respondentes declaram não serem esses os principais problemas.

\subsection{Análise dos indicadores de desempenho logístico na RMSP}

As Tabelas 6 e 7 apresentam as medidas descritivas dos indicadores de desempenho logístico que, na visão do Operador logístico e Transportador, respectivamente, representam o maior ou menor índice. Os indicadores de índices mais baixos são indicados por "Zero" e os de maior índice por "Um.

Pelas medidas descritivas apresentadas nas Tabelas 6 e 7, nota-se que os operadores logísticos e as transportado- ras têm opiniões semelhantes quanto ao desempenho logístico frente aos indicadores que possuem o melhor resultado. Ou seja, o "atendimento das entregas urgentes", "tempo de entrega na última semana do mês" e "cumprimento do prazo de entrega" receberam notas médias maiores que 5; até 50\% dos respondentes $(\mathrm{Md})$ atribuíram notas 5,5 ou $6,75 \%$ atribuíram notas acima de 4,75 (Q1), sendo destes, 25\% (Q3) atribuíram notas 6 ou 7 a eles; ou seja, representam ótimo desempenho logístico. Neste sentido, o "baixo índice de avarias nas cargas" foi declarado como bom desempenho pelas transportadoras (com $75 \%$ das notas maiores ou iguais a 4 - Q1). Por outro lado, as análises estatísticas denotam os piores valores dos indicadores de desempenho logísticos "disponibilidade de veículos", "devoluções de mercadorias" e "confirmação de entrega em tempo real" para os operadores logísticos. Para esses indicadores, cerca de $25 \%$ atribuíram notas 2 ou 3 (Q1) e 50\% notas menores ou iguais a 4,5 .

\section{DISCUSSÃO DOS RESULTADOS E CONCLUSÕES}

Nesta pesquisa os operadores logísticos são empresas de grande porte, com localização predominantemente no Estado de São Paulo e que atendem à RMSP com frota de veículos mais leves e bem distribuídas em pequenos varejos, além de terceirizarem a maior parte dos serviços de entregas. Um aspecto interessante é que operadores logísticos atuam fortemente em outras regiões do Estado. Esses fatos podem explicar a dificuldade para contratar veículos com placas de numeração diversificada para atender o rodízio de transportadores e também garantir disponibilidade excedente de veículos por parte da transportadora para a entrega concentrada de mercadorias no final do mês (como comentado pelos entrevistados), que auxilia a gerar longas filas. Por outro lado, as transportadoras são normalmente empresas de porte pequeno a médio, concentradas dentro da RMSP e, portanto, tendem a ter maior conhecimento dos problemas locais. Consequentemente, as transportadoras podem oferecer um serviço de qualidade aos seus clientes (os operadores logísticos) e, também, perceber mais acentuadamente os problemas por atuar na última milha. Como exemplo, "a falta de comprometimento no recebimento" é percebida como problema mais para o Transportador do que para o Operador logístico. Um fato que chama a atenção é a Lei dos motoristas que parece não ser problema para as transportadoras. Isso leva a crer que a lei possa ser de fácil ajuste, dado que as entregas têm características locais.

Tanto para os operadores logísticos quanto para as transportadoras, o "congestionamento" e o "roubo de carga" são problemas. Esse resultado também foi relatado pelos entrevistados. Tal percepção acende uma luz vermelha aos olhos dos gestores públicos que necessitam considerar as opiniões das empresas envolvidas no transporte urbano de carga no planejamento da RMSP. A falta de uma política conjunta é ressaltada também por Lindholm e Behrends (2012) e Lindholm (2013).

Os regulamentos referentes ao atendimento de emissões de gases de efeito estufa não são considerados problema para ambas as categorias. Com base nesses resultados e nas entrevistas com alguns representantes das transportadoras, é possível afirmar três situações que ocorrem na prática das empresas: (a) ainda não há pressão por parte da 
VIEIRA, J.G.V.; CARVALHO, C.D.; YOSHIZAKI H.T.Y.

Tabela 5. Medidas descritivas dos indicadores de desempenho logístico para o Operador Logístico

\begin{tabular}{|c|c|c|c|c|c|}
\hline Indicador & Destaque & Média & Q1 & Md & Q3 \\
\hline Atendimento das entregas urgentes & 1 & 5,46 & 5 & 6 & 6 \\
\hline Baixo tempo de entrega na última semana do mês & 1 & 5,25 & 4,75 & 6 & 7 \\
\hline Cumprimento do prazo de entrega & 1 & 5,21 & 4,75 & 5,5 & 6 \\
\hline OTIF & & 5,00 & 5 & 5 & 6 \\
\hline Baixo índice de avaria nas cargas & & 4,88 & 3 & 5 & 6 \\
\hline Baixo índice de roubo de carga & & 4,58 & 3 & 5 & 6 \\
\hline Baixo índice de extravio de carga & & 4,50 & 3 & 5 & 6 \\
\hline Trajeto com menor percurso & & 4,50 & 3 & 5 & 6 \\
\hline As entregas são sempre sem erro & & 4,46 & 3 & 5 & 5 \\
\hline Alta disponibilidade de veículos & 0 & 4,13 & 3 & 4 & 5 \\
\hline Baixo índice de devoluções & 0 & 3,96 & 2 & 4 & 5 \\
\hline Confirmação de entrega em tempo real & 0 & 3,96 & 2 & 4,5 & 5 \\
\hline
\end{tabular}

Tabela 6. Medidas descritivas dos indicadores de desempenho logístico para o Transportador

\begin{tabular}{lccccc}
\hline Indicadores & Destaque & Média & Q1 & Md & Q3 \\
\hline Atendimento das entregas urgentes & 1 & 5,49 & 4,5 & 6 & 7 \\
Baixo tempo de entrega na última semana do mês & 1 & 5,46 & 5 & 6 & 7 \\
Cumprimento do prazo de entrega & 1 & 5,24 & 4,5 & 6 & 6,5 \\
Baixo índice de avaria nas cargas & $\mathrm{I}$ & 5,14 & 4 & 5 & 7 \\
Baixo índice de extravio de carga & & 5,04 & 3,5 & 6 & 7 \\
OTIF & & 4,79 & 3 & 5 & 6 \\
Confirmação de entrega em tempo real & & 4,72 & 3 & 5 & 6.5 \\
As entregas são sempre sem erro & & 4,60 & 3 & 5 & 6 \\
Baixo índice de devoluções & 4,56 & 3 & 5 & 6 \\
Baixo índice de roubo de carga & & 4,47 & 2,5 & 5 & 6 \\
Alta disponibilidade de veículos & & 4,29 & 3 & 5 & 6 \\
Trajeto com menor percurso & & 4,23 & 3 & 5 & 6 \\
\hline
\end{tabular}

sociedade, do Governo e das empresas (embarcadoras e varejistas) suficiente para acelerar o processo de controle/regulação de emissão de gases na RMSP, já que cada cidade da região tem seu próprio regulamento; (b) a adequação às leis atuais não representam um elevado aporte financeiro que leve as empresas à troca de veículos e ou manutenção preventiva de elevado custo; (c) as empresas já atendem a todas as condições impostas.

No entanto, esta é ainda uma questão a ser investigada por meio de um levantamento de dados exclusivo com as transportadoras que são as empresas que estão mais diretamente envolvidas com esta questão.

Quanto aos indicadores, o operador logístico parece ter dificuldade de lidar com "confirmação de entrega em tempo real" e com a "alta taxa de devolução de mercadorias" mais do que o transportador. Ou seja, esse fato confirma a dificuldade de o operador logístico atender à diversidade de clientes, destinatários e regiões. Por outro lado, o transportador parece revelar o pior desempenho para "trajeto com menor percurso" e "roubo de carga". Quanto ao roubo de carga, esse resultado pode revelar menor ocorrência dentro da RMSP, já que $97 \%$ dessas empresas atuam localmente.

Por outro lado, é estranho o indicador "trajeto com menor percurso" apresentar o pior desempenho logístico para os transportadores, pois espera-se que conheçam bem os locais de entrega, as regras e dificuldades encontradas para estacionar e descarregar as cargas. Talvez os operadores logísticos possam atender os consumidores por meio de diferentes tipos de veículos. O pior desempenho deste indicador também pode revelar pouco uso de sistema de roteirização e uso de rotas alternativas para desviar de congestionamentos. Assim, o atributo "congestionamento", levantado como um dos principais problemas, pode se revelar como um dos principais atributos que impactam o desempenho das transportadoras.

Os prestadores de serviço revelaram um baixo desempenho logístico com relação à "disponibilidade de veículo". Este resultado sugere que os atributos logísticos como, por exemplo, "acúmulo de entregas na última semana do mês", "vias de acesso estreitas para entrega" e "necessidade de diferentes tipos de veículos" são os que mais podem impactar a disponibilidade de veículo. Quanto maior a variabilidade da demanda dos clientes e urgência na entrega de mercadorias, menor será a disponibilidade de veículos e maior custo maior custo na contratação de serviços diversificados, manutenção de veículos, e treinamento de motoristas para ambos, o transportador e o operador logístico.

Este trabalho contribui para a literatura, pois investigou a relevância dos problemas enfrentados pelas empresas responsáveis pela entrega de mercadorias na RMSP. Adicionalmente, os indicadores de desempenho foram objeto de pesquisa e mostraram que há indícios de que os maiores 
problemas enfrentados pelas empresas impactam negativamente no seu desempenho logístico. Logo, esta pesquisa auxilia as empresas, pois mostra a diversidade de problemas que elas enfrentam para o atendimento às entregas de mercadorias na RMSP e quais indicadores de desempenho logístico precisam ser melhorados.

Como limitações da pesquisa destacam-se a análise univariada de dados e o tamanho da amostra. Neste sentido, análises mais robustas por meio de técnicas multivariadas poderiam ser futuramente aplicadas a fim de evitar a possibilidade de ocorrência de erro tipo 1, pois essas variáveis podem estar altamente correlacionadas. Logo, esta pesquisa faz um levantamento dos problemas e indicadores de desempenho e apresenta uma análise individual de cada variável. Certamente, técnicas mais robustas, como Modelos de Equações Estruturais, poderão ser úteis na medida em que avaliam as dependências causais entre os indicadores de desempenho logístico (em si) e entre estes e os problemas enfrentados pelas empresas na RMSP. A abordagem de equações estruturais é interessante para este tipo de aplicação porque apresenta, por exemplo, o quanto uma restrição, seja esta regulatória ou falta de colaboração, afeta efetivamente os indicadores de desempenho logístico. Logo, esta abordagem permitiria quantificar este impacto e dar a visão das relações de causa e feito, e não somente revelar indícios de que os problemas avaliados separadamente podem ou não afetar certos indicadores.

Ressalta-se que este artigo faz parte de um projeto de pesquisa sobre a RMSP que pode ter grande relevância para fornecer diretrizes para que o planejamento urbano de carga possa ser incluído de forma sistemática nas políticas públicas. Assim, espera-se, no futuro, avaliar os problemas apresentados pelas empresas desta pesquisa em conjunto com os decisores de planejamento de transporte na RMSP. Esta avaliação poderá, ainda, ajudar na futura elaboração de regras para a mobilidade urbana de carga e na mitigação desses problemas identificados na distribuição de carga na RMSP.

\section{AGRADECIMENTOS}

Os autores agradecem ao CNPq pelo financiamento parcial desta pesquisa (Projeto número: 2480-13-1; 301140/2013-8).

\section{REFERÊNCIAS}

Allen, J.; Anderson, S.; Browne, M.; Jones, P. (2000). A framework for considering policies to encourage sustainable urban freight traffic and goods/service flows. In: Current goods and service operations in urban areas. London University of Westminster.

Allen, J., Browne, M. 3 Cherrett, T. (2012). Investigating relationships between road freight transport, facility location, logistics management and urban form. Journal of Transport Geography, v.24, p.45-57. DOI:10.1016/j.jtrangeo.2012.06.010.

Anand, N., Quak, H., Duin, R. Van e Tavasszy, L. (2012). City logistics modeling efforts: Trends and gaps $-\mathrm{A}$ review. Procedia - Social and Behavioral Sciences, v.39, p.102-115.

DOI:10.1016/j.sbspro.2012.03.094

Antún, J. P., Lozano, A., Alarcón, R., Granados, F. e Guarneros, L. (2010). The physical distribution of goods in a megalopolis:
Strategies for policies on the location of logistics facilities within the Central Region of Mexico. Procedia - Social and Behavioral Sciences, v.2, n.3, p.6130-6140.

DOI:10.1016/j.sbspro.2010.04.025.

Ballantyne, E. E. F., Lindholm, M. e Whiteing, A. (2013). A comparative study of urban freight transport planning: addressing stakeholder needs. Journal of Transport Geography, v.32, p.93101. DOI:10.1016/j.jtrangeo.2013.08.013.

Behrends, S., Lindholm, M. e Woxenius, J. (2008). The impact of urban freight transport: A definition of sustainability from an actor's perspective. Transportation Planning and Technology, v.31, n.6, p.693-713. Doi:10.1080/03081060802493247

Browne, M.; Allen, J.; Nemoto, T.; Patier, D.; Visser, J. (Jan2012). Reducing social and environmental impacts of urban freight transport: A review of some major cities. Procedia Social and behavioural Sciences, v.39, p.19-33.

DOI:10.1016/j.sbspro.2012.03.088.

Castro, J. T., Kuse, H. e Hyodo, T. (2003). A study on the impact and effectiveness of the truck ban scheme in metro Manila. Journal of the Eastern Asia Society for Transportation Studies, v.5, p. 2177-2192.

Cherrett, T., Allen, J., McLeod, F., Maynard, S., Hickford, A. e Browne, M. (2012). Understanding urban freight activity - key issues for freight planning. Journal of Transport Geography, v.24, p.22-32. DOI:10.1016/j.jtrangeo.2012.05.008.

Costabile, F. e Allegrini, I. (2008). A new approach to link transport emissions and air quality: An intelligent transport system based on the control of traffic air pollution.

Environmental Modelling \& Software, v.23, n.3, p.258-267. DOI:10.1016/j.envsoft.2007.03.001.

Dablanc,L. (1997). Entre police at modeling: I'action publique sur le transport de merchadises um ville: Le cas des modeling de Paris et New York. Laboratoire Doctorat de Techniques, Territoires et 1'Ecole nationale des ponts et epécialité: transports Sociétés modelling.

Dablanc, L. (2007). Goods Transport in Large European Cities: Difficult to Organize, Difficult to Modernize. Transportation Research Part A: Policy and Practice, v.41, n.3, p.280-285. DOI:10.1016/j.tra.2006.05.005

De Oliveira, L. K. (2007). Modelagem para avaliar a viabilidade de implantação de um sistema de distribuição de pequenas encomendas dentro dos conceitos de city logistics. Tese (Doutorado), PPGEP, Florianópolis, SC.

De Oliveira, L. K., Braga, A. de S. e Abreu, B. R. A. (2010). Relevant attributes in overnight goods delivery: researches, transporters, and retailers preferences in urban distribution. Anais... 12Th WCTR. Lisbon: Portugal.

De Vasconcellos, E., A. (2005). Urban change, mobility and transport in São Paulo: three decades, three cities. Transport Policy, v.12, p.91-104. DOI: 10.1016/j.tranpol.2004.12.001.

Dutra, N. G. D. S. (2004). O enforque de city logistics na distribuição urbana de encomendas. Tese (Doutorado), Programa de Pós-Graduação em Engenharia de Produção da Universidade Federal de Santa Catarina.

Forza, C. (2002). Survey research in operations management: a process-based perspective. International Journal of Operations \& Production Management, v.22, n.2, p.152-194. DOI:

10.1108/01443570210414310. 
Hesse, M e; Rodrigue, J. P. (2004). The transport geography of logistics and freight distribution. Journal of Transport Geography, v.12, n.3, p.171-184.

DOI:10.1016/j.jtrangeo.2003.12.004

IPEA (2015). Governança Metropoliatana no Brasil. Relatório de Pesqusia. 82p. Acesso em 02/05/2016. Disponível em http://www.ipea.gov.br/redeipea/images/pdfs/governanca_metro politana/150730_relatorio_arranjos_saopaulo.pdf.

Ljungberg, D. e Gebresenbet, G. (2005). Mapping out the potential for coordinated goods distribution in the city centers: The case of Uppsal. International Journal of Transport Management, v.2, n 2005, p.161-172. DOI: 10.1016/j.ijtm.2005.07.001.

Lindholm, M. e Behrends, S. (2012). Challenges in urban freight transport planning - a review in the Baltic Sea Region. Journal of Transport Geography, v.22, p.129-136. DOI:10.1016/j.jtrangeo.2012.01.001.

Lindholm, M. (2013). Urban freight transport from a local authority perspective - a literature review. European Transport $\backslash$ Trasporti Europei, v.54, p.1-37.

Lu, C.-S. (2003). The impact of carrier service attributes on shipper-carrier partnering relationships: a shipper's perspective. Transportation Research Part E: Logistics and Transportation Review, v.39, n.5, p.399-415. DOI: 10.1016/S13665545(03)00015-2.

Moreira, C. M. (2012). Metodologia para obtenção de dados e informações em logística urbana - estudo de caso da região metropolitana de Belo Horizonte. Dissertação (Mestrado) em Geotecnia e Transportes, Escola de Engenharia, Universidade Federal de Minas Gerais. $134 \mathrm{f}$.

Munuzuri, J., Larraneta, J., Onieva, L. e Cortes, P. (2005). Solutions applicable by local administrations for urban logistics improvement. Cities, v.22, n.1, p.15-28.

DOI:10.1016/j.cities.2004.10.003.

Muñuzuri, J., Cortés, P., Guadix, J. e Onieva, L. (2012). City logistics in Spain: Why it might never work. Cities, v.29, n.2, p.133-141. DOI:10.1016/j.cities.2011.03.004.

Ogden, K. W. (1992). Urban Goods Movement: A Guide to Policy and Planning. Inglaterra: Editora Ashgate.

Pires, S. (2011). Gestão da cadeia de suprimentos: conceitos, estratégias, práticas e casos. $2^{\mathrm{a}}$ edição. São Paulo: Editora Atlas.

Sanches Junior, P. F. (2008). Logística de Carga Urbana: uma análise da realidade brasileira. Tese (Doutorado), UNICAMP, Campinas, SP.

Sebrae. (2013). Sebrae. Critérios de classificação de empresas: EI - ME - EPP. Disponível em: <http://www.sebraesc.com.br/leis/default.asp?vcdtexto=4154>. Acesso em: 12 ago. 2013.

Stathopoulos, A., Valeri, E. e Marcucci, E. (2012). Stakeholder reactions to urban freight policy innovation. Journal of Transport Geography, v.22, p.34-45. DOIi:10.1016/j.jtrangeo.2011.11.017.

Stefansson, G. (2006). Collaborative logistics management and the role of third-party service providers. International Journal of Physical Distribution \& Logistics Management, v.36, n.2, p.7692. DOI:10.1108/09600030610656413.
Tacla, D., Lima Junior, O. F. e Botter, R. C. (2006). A collaborative transportation proposal for urban deliveries: costs and environmental savings. Urban Transport XII. Urban Transport and the Environment in the 21st Century. Anais... Prage, Czech Republic.

Taniguchi, E. e Thompson, R. G. (2002). Modelling city logistics, Transportation Research, p. 45-51.

Transvias. Cadastro de prestadores de serviço. Disponível em: $\langle$ http://www.transvias.com.br/1/25/transportadoras/Sao-Paulo>. Acesso em: 22 abr. 2015.

Vieira, J. G.V., Yoshizaki, H. e Ho, L. (2009). Collaboration intensity in the Brazilian supermarket retail chain. Supply Chain Management, v.1, p.11-21. DOI:10.1108/13598540910927269.

Vieira, J. G.V., Yoshizaki, H. e Ho, L. (2015). The effects of collaboration on logistical performance and transaction costs. International Journal of Business Science \& Applied Management, v.10, n.1, p.1-14.

Vivaldini, M. e Pires, S. R. I. (2012). Gestão da demanda, colaboração e CPFR (Collaborative Planning, Forecasting, and Replenishment): um estudo em empresas de alimentação, Sistema e Gestão Revista Eletrônica, v.7, p.312-323. DOI: 10.7177/sg.2012.v7.n3.a2.

Zioni, S. M. (2009). Espaços de carga na região metropolitana de São Paulo. Tese (Doutorado), Faculdade de Arquitetura e Urbanismo da Universidade de São Paulo, São Paulo, SP. 
